\title{
Macrofauna del suelo como indicador biológico del estado de conservación en sistemas agroforestales del sector el Choclino en San Martín - Perú
}

Soil macrofauna as biological indicator of the state of conservation in agroforestal systems of the sector el Choclino in San Martín - Peru

${ }^{1}$ Shirley Ornella Castillo Pérez, ${ }^{\mathrm{a}}$ Manuel Ñique Álvarez ${ }^{\mathrm{b}}$

\section{RESUMEN}

En la actualidad la identificación de los indicadores biológicos de la calidad del suelo es un problema universal, por lo que la evaluación de la macrofauna edáfica es apropiada para investigar el estado de conservación/perturbación del suelo. En tal sentido, la investigación planteó como objetivo: evaluar la macrofauna edáfica como indicador biológico de conservación del suelo en sistemas agroforestales en el sector Choclino - San Martín. El estudio se realizó en el Instituto de Cultivos Tropicales, distrito de la Banda de Shilcayo, provincia de San Martín, región de San Martin; se empleó dos sistemas agroforestales el tradicional y el nativo. El muestreo de macrofauna se realizó de acuerdo a la metodología T.S.B.F. propuesta por Anderson e Ingram, (1993); se realizó el análisis taxonómico, indicadores agregados, parámetros ecológicos y se determinó el análisis estadístico del índice de diversidad de Shannon Wiener en los dos sistemas agroforestales. Los resultados muestran que el INAS tiene mayor densidad y biomasa de macrofauna que el ITAS; las curvas de dominancia $\mathrm{ABC}$ presentaron una moderada perturbación; el atributo comunitario en el INAS tuvo una diversidad alta (2.11439) y en ITAS medio (1.87439); el índice de equidad en ambos sistemas agroforestales tiene un valor medio; los dos sistemas agroforestales son muy significativamente distintos $(\mathrm{P}<0.001)$ en términos de diversidad de macrofauna del suelo.

Palabras clave: diversidad, equidad, densidad, biomasa, macrofauna.

\begin{abstract}
At present the identification of biological indicators of soil quality is a universal problem, so the evaluation of the edaphic macrofauna is appropriate to investigate the state of conservation / disturbance of the soil. In this sense, the objective of the research was to evaluate the edaphic macrofauna as a biological indicator of soil conservation in agroforestry systems in the Choclino - San Martín sector. The study was carried out at the Institute of Tropical Crops, district of the Banda de Shilcayo, province of San Martin, region of San Martin; two traditional and native agroforestry systems were used. Sampling of macrofauna was done according to the methodology T.S.B.F. proposed by Anderson and Ingram, (1993); the taxonomic analysis, aggregate indicators, ecological parameters and the statistical analysis of Shannon Wiener diversity indices in the two agroforestry systems were


determined. The results show that INAS has higher density and macrofauna biomass than ITAS; the ABC dominance curves showed a moderate perturbation; the community attributes in the INAS had a high diversity (2.11439) and average ITAS (1.87439); the equitability index in both agroforestry systems has an average value; the two agroforestry systems are very significantly different $(\mathrm{P}<0.001)$ in terms of soil macrofauna diversity.

Key words: diversity, equitability, density, biomass, macrofauna.

${ }^{1}$ Universidad Nacional Agraria de la Selva.

${ }^{2}$ Universidad Nacional de Cañete.

${ }^{\mathrm{a}}$ Ingeniera Ambiental, ${ }^{\mathrm{b}}$ Biólogo 


\section{INTRODUCIÓN}

La macrofauna son aquellos organismos macro invertebrados que componen la fracción viva y orgánica del suelo y se encuentran comprendidas entre $2 \mathrm{~mm}$ a 20 mm de longitud, se mueven activamente en el suelo y pueden elaborar galerías en las cuales viven (Ramirez, 2005) y abarca Anélidos (lombrices) Coleópteros (escarabajos) Himenópteras- Formícidas (hormigas) Isópteras (termitas) y estados adultos e inmaduros de otros artrópodos edaficolas (Decaens et al., 1994).

Los invertebrados que integran la macrofauna del suelo manifiestan algunos de estos rasgos, lo cual justifica su utilización como indicadores biológicos. Entre ellos se pueden señalar: la ventaja de su diversificación taxonómica y ecológica, sus hábitos relativamente sedentarios, la presencia a lo largo del año, y la posibilidad de que sean manipulados e identificados (tratamiento taxonómico). También su corto período entre generaciones permite una rápida respuesta poblacional a los cambios ambientales, y su alta densidad y capacidad de reproducción posibilitan un muestreo intensivo, sin que ello provoque desequilibrio en la comunidad. Otras características son: su importancia funcional en los ecosistemas y una respuesta aparentemente previsible ante las perturbaciones (Brown, 1997; McGeoch, 1998; Jones y Eggleton, 2000; McGeoch, van Rensburg y Botes, 2002; citados por Cabrera, 2012). Desde el punto de vista biológico, en la evaluación del estado de conservación del suelo y del ecosistema se puede tomar en cuenta la macrofauna edáfica, que también intervienen en la trituración de los restos vegetales y algunos funcionan como depredadores de animales vivos de la macrofauna y la mesofauna edáfica (Robaina et al., 2011; citados por Cabrera, 2012).

En la actualidad la identificación de los indicadores de la calidad del suelo es un problema universal; por lo general, se han utilizado un grupo de variables para predecir la salud del suelo, a partir del estado de sus propiedades físicas, químicas y/o biológicas (Cabrera, 2012). En ese sentido Martínez (2012), menciona que el método de comparación Abundancia-Biomasa (ABC) fue inicialmente propuesto por Warwick (1986), como una técnica para detectar perturbaciones (principalmente efectos de contaminación) en comunidades de invertebrados bentónicos, comparando la dominancia en términos de abundancia y biomasa. Las curvas $\mathrm{ABC}$ presentan un marco teórico en la teoría evolutiva clásica de la selección r y k. En estado no perturbado, la comunidad se supone que es dominado por especies con estrategia $\mathrm{k}$ (crecimiento lento, grandes, y maduración tardía), la curva de la biomasa se encuentra por encima de la curva de la abundancia. Cuando se presenta un incremento gradual de perturbación, las especies de crecimiento lento no dan abasto, y el sistema pasa a ser dominado 
cada vez más por especies con estrategias r (crecimiento rápido, pequeñas, oportunistas), causando que la curva de biomasa pase a estar por debajo de la curva de abundancia.

El diagnóstico depende del valor obtenido del estadístico W de las curvas $\mathrm{K}$ dominancia (Lambshead et al., 1983, Warwick y Clarke, 1994; citados por Martínez, 2012) para la abundancia. La diferencia entre las dos curvas se obtiene mediante el estadístico $\mathrm{W}$, que representa el área entre ellas. El índice es negativo para situaciones caracterizadas por un fuerte stress, cercano a 0 tendería a presentar un moderado stress y positivo a un mínimo de stress (Meire y Dereu, 1990; Coeck et al., 1993; citados por Martínez, 2012). El signo negativo indica que la curva de la biomasa está por debajo de la curva de abundancia y sugiere una comunidad perturbada. Una ventaja del método es que, a partir de un conjunto de datos adecuados para cada área o tiempo, permita conocer el estado de la comunidad que se está evaluando, sin la necesidad de un control espacial o temporal contra el cual se va a comparar el índice obtenido, ya que la biomasa es comparada con la abundancia para el mismo tiempo y lugar (Martínez, 2012).

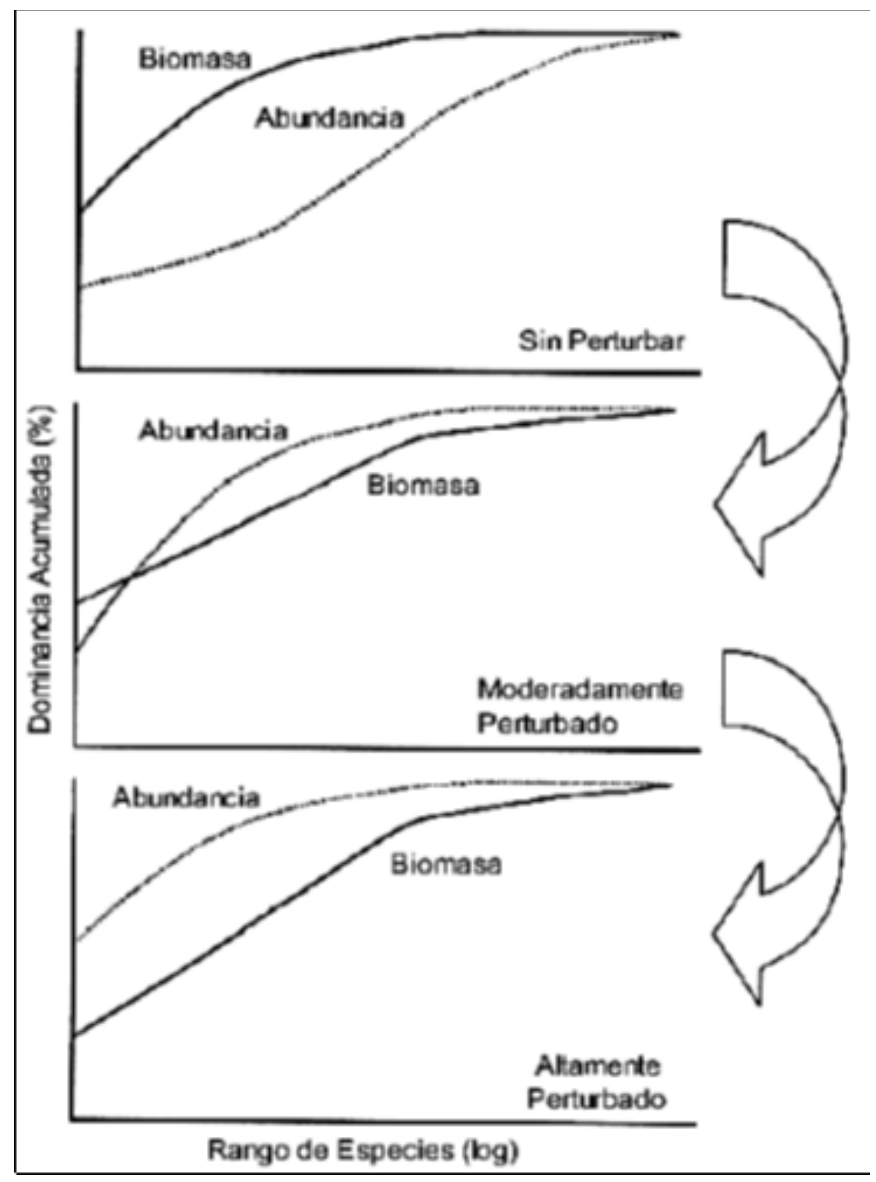

Figura 1. Curvas ABC mostrando el patrón en la abundancia y biomasa de asociaciones sin perturbación, moderadamente perturbada y altamente perturbada.

Fuente: Martínez (2012). 
Con respecto al análisis de 1 a biodiversidad del suelo, Odum (1972), menciona que el índice de Shannon Wiener mide el grado de incertidumbre que existe para predecir la especie a la cual pertenece un individuo extraído aleatoriamente de la comunidad para un numero dado de especies e individuos, la función tendrá valor mínimo cuando todos los individuos pertenecen a una especie y un valor máximo cuando todas las especies tengan la misma cantidad de individuos. Una característica de Shannon Wiener es su sensibilidad a los cambios en la abundancia de las especies raras. Y Ñique (2010), menciona que una característica de Shannon Wienner es su sensibilidad a los cambios en la abundancia de las especies raras; por ello es aplicable en los estudios de conservación de la naturaleza. Para su cálculo de la diversidad se puede utilizar diversos logaritmos: Ln, $\log 10$ y $\log 2$, correspondiéndole las siguientes unidades: Ln: bels naturales/individuo o nats/individuo; $\log 10$ : decits/individuo; Log2: bits/individuo. Los valores que se obtiene con este índice generalmente están entre 1.5 y 3.5 y raramente sobrepasa a 4.5 (Magurran, 1989).

Del mismo modo Ramírez (2006), señala los valores que toma el índice de Shannon-Wiener, donde indica que los valores que se encuentren dentro del rango de $0-1$ presentan diversidad muy baja, mayor de $1-1.8$ presentan diversidad baja, mayor de $1.8-2.1$ presentan diversidad media, mayor de 2.1
-2.3 presentan diversidad alta y mayores a 2.3 presentan diversidad muy alta. Y Magurran (1989) menciona que el valor de equidad se sitúa entre 0 y 1.0 , donde 1.0 representa una situación en la que todas las especies son igualmente abundantes. Barrios (1999) citado por Campos (2010) observo que la calidad de la hojarasca tiene mayor injerencia que su cantidad sobre la riqueza de especies de la macrofauna. El coeficiente de variación de la biomasa es de 0.85 esto quiere decir que existe una baja dispersión entre los valores de los diferentes sistemas de uso.

El objetivo general del presente estudio es determinar las características de la macrofauna del suelo como indicador biológico del estado de conservación en sistemas agroforestales del sector el Choclino en San Martín - Perú. Estableciéndose los objetivos específicos: Estimar y analizar las variaciones de biomasa y densidad de taxones de macrofauna identificadas a través de indicadores agregados (curvas abundancia-biomasa $\mathrm{ABC}$ ); determinar y analizar los atributos comunitarios (diversidad y equidad) entre los sistemas agroforestales nativo y tradicional; $\mathrm{y}$ comprobar las diferencias significativas de diversidad entre los dos sistemas agroforestales.

\section{MATERIALES Y MÉTODOS}

La investigación se inició en el mes de agosto del 2014, desarrollándose en dos 
(02) parcelas con diferentes sistemas agroforestales (Nativo y Tradicional) de la estación experimental "El Choclino" del Instituto de Cultivos Tropicales (ICT), ubicado en el departamento de San Martín, provincia de San Martín, distrito de la Banda de Shilcayo, en el sector El Choclino (coordenadas UTM: Este: 352727, Norte: 9283870 y a una altitud de entre 500-530 m.s.n.m.

Respecto a sus características ambientales, la zona corresponde a la formación de bosque húmedo Premontano Tropical (bh-PT); presenta las siguientes características climatológicas: humedad de $77 \%$, temperatura media anual $27^{\circ} \mathrm{C}$ y una precipitación promedio de 936 y 1800 mm(ONERN, 1976).

\section{DESCRIPCIÓN DE LOS DOS SITIOS DE MUESTREO:}

\section{Sistema agroforestal nativo (INAS)}

Es un bosque secundario de 25 años de edad, existiendo dentro su composición florística diversidad de especies con propiedades medicinales ${ }_{2}$ maderables, lianas, leña, entre otros. Para el establecimiento del cacao, se realizó un raleo selectivo de árboles (rozo) de una densa vegetación que sirve de sombra permanente para la plantación de cacao a una alta densidad.

\section{Sistema agroforestal tradicional mejorado(ITAS)}

Es un bosque secundario de 25 años de edad, se realizó rozo, tumba y quema antes de establecer la plantación de cacao.
El mismo proceso que realizan los agricultores para el establecimiento de cacao, área en la cual se sembró frijol, maíz, yuca y como sombra temporal plátano. También se sembró Inga sp., como sombra permanente.

\section{MATERIALES Y EQUIPOS}

Durante el trabajo en campo, la obtención de las muestras de suelo en los sistemas agroforestales se utilizaron libreta de campo, stickers para codificar, rafia, cinta maskingtape, plumón indeleble, caja tecnopor, bolsas de polietileno (21x24), bolsas de polipropileno de $1 \mathrm{~kg}$ (10x12), termómetro de suelo, palanas, machetes, cinta métrica, muestreador de metal (25 x 25 x 30), G.P.S., cámara digital fotográfica.

Para el trabajo en el laboratorio, la extracción de la macrofauna de las muestras de suelo recolectadas se utilizaron guantes quirúrgicos, viales de vidrio, pinzas, pipetas de $10 \mathrm{ml}$, probeta de $250 \mathrm{ml}$; asi como también equipos de laboratorio y reactivos, tales como microscopio, estereoscopio, balanza de precisión, lámpara LED, lupa, alcohol $70 \%$, glicerina $5 \%$, formol $5 \%$ y agua destilada.

\section{METODOLOGÍA}

\section{Muestreo de la macrofauna del suelo}

Los suelos se muestrearon con el método del azar, con la ayuda de un guía de metal obtuvo nueve (9) monolitos por sistema a agroforestal con cuatro (4) submuestras 
por monolito. El monolito tuvo la siguiente dimensión: 25 × 25 × $30 \mathrm{~cm}$ conforme se muestra en la figura 2.

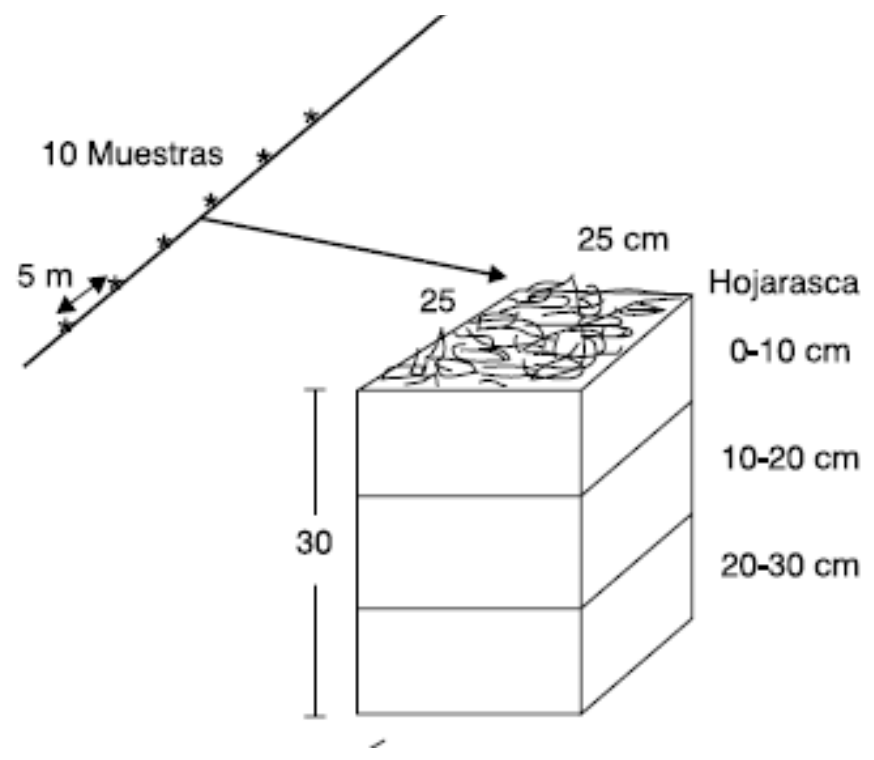

Figura 2. Corte y División de monolito

Fuente: Adaptado de Pashanasi (2001)

El método de muestreo e interpretación de resultados se basó en la metodología de Tropical Soil Biology and Fertility (TSBF) (Anderson \& Ingram, 1993). Los nueve (9) monolitos y/o muestras fueron tomadas en cuatro (4) diferentes niveles de profundidad (estrato): hojarasca, 0-10 cm, 10-20 cm y 20-30 cm; los organismos fueron identificados por unidades taxonómicas (familia y órdenes) en el laboratorio de entomología de la Universidad Nacional Agraria de la Selva - UNAS. De cada estrato $(10 \mathrm{~cm}) \mathrm{se}$ colectaron la macrofauna y se depositaron en soluciones de alcohol al 70\%, para insectos de cuerpo endurecido en glicerina al $5 \%$ y en formol del 5\% las larvas e insectos de cuerpo no endurecido (Decaens et al., 1994).

\section{Análisis taxonómico}

El conteo de la macrofauna se realizó después de recolectado las muestras con el monolito de metal. De los organismos colectados; se cuantificó la biomasa ( $\mathrm{g} / \mathrm{m} 2$ ) y densidad (individuos $/ \mathrm{m} 2$ ) por medio de estereoscopio y una balanza de precisión. Los valores de biomasa fueron multiplicados por un valor de corrección (19\% para las lombrices, 9\% hormigas, $11 \%$ escarabajos, $6 \%$ arañas y $13 \%$ para el resto de macro invertebrados) debido a la pérdida de peso durante la fijación en alcohol, glicerina y formol (Decaens et al. 1994).

\section{Curvas Abundancia- Biomasa ABC}

Para construir la curva, se toma como eje $\mathrm{X}$ (escala logarítmica) los rangos de abundancia de las especies (rango incluye a las especies más abundantes y como eje Y (escala acumulativa) el número de especies que entran en cada rango. La diferencia entre las dos curvas se obtiene mediante el estadístico $\mathrm{W}$, que representa 
el área entre ellas y asociaciones al índice $\mathrm{ABC}$, se calculó por medio de la siguiente formula (Warwick y Clarke, 1994; citados por Martínez, 2012).

$$
W=\frac{\sum_{\mathrm{t}=1}\left(B_{i}-A_{i}\right)}{[50(S-1)]}
$$

Dónde:

$\mathrm{W}=$ Suma estandarizada de las diferencias entre cada par de especies de la biomasa acumulada y la abundancia acumulada, valor ordenado en forma decreciente.

$\mathrm{Bi}=$ Porcentaje de dominancia de la especie i (rango de alta a baja biomasa).

$\mathrm{Ai}=$ Rango de las especies más abundantes a las menos.

$\mathrm{S}=$ Total del número de especies.

\section{ANÁLISIS DE PARÁMETROS ECOLÓGICOS}

\section{Diversidad}

Se asume que los individuos son seleccionados al azar y que todas las especies están representadas en la muestra. Adquiere valores entre cero, cuando hay una sola especie, y el logaritmo de S, cuando todas las especies están representadas por el mismo número de individuos (Magurran, 1989).

Se consideró el número y la proporción de individuos por orden en cada uno de los monolitos. Para su estimación se utilizó la expresión matemática propuesta por Shannon-Wiener.

$$
H^{\prime}=-\Sigma p_{\mathrm{i}} \ln p_{\mathrm{i}} \quad p_{\mathrm{i}}={ }^{\mathrm{n}_{\mathrm{i}}} / \mathrm{N}
$$

Donde:

$\mathrm{H}^{\prime}=$ Diversidad de especies pi $=$ Proporción de individuos de la especie i respecto al total de individuos. $\mathrm{N}=$ Número de todos los individuos de todas las especies $\mathrm{ni}=$ Número de individuos de la especie $\mathrm{i}$

\section{Equidad}

Mide la proporción de la diversidad observada con relación a la máxima diversidad esperada. Su valor va de 0 a 0.1 , de forma que 0.1 corresponde a situaciones donde todas las especies son igualmente abundantes. Se expresa como sigue (Moreno, 2001):

$$
\mathrm{H}_{\max }=\mathrm{Ln} S \quad \mathrm{E}=\frac{\mathrm{H}^{\prime}}{\mathrm{H}_{\max }}
$$

Donde:

$S=$ número de especies

\subsection{Variables a evaluar}

Para poder evaluar la macrofauna como indicador biológico en los diferentes tipos de uso de tierra, se evaluó los componentes biológicos del suelo que se muestran en el tabla 1.

Tabla 1. Indicadores biológicos del suelo

\begin{tabular}{ll}
\hline Indicadores biológicos & Método de determinación \\
\hline Densidad de fauna de suelo & Método directo por conteo \\
Biomasa de la fauna de suelo & Método directo, pesaje \\
Indicadores agregados & Curva abundanci a- biomasa (ABC) \\
Diversidad de especies & Método cálculo de los Índices de Shannon \\
& Wiener y Equidad. \\
\hline
\end{tabular}

Fuente: Warwick y Clarke, 1994, Magurran, 1988 y Moreno, 2001 citados por Martínez, 2012. 


\section{Análisis estadístico del índice de diversidad}

Según Magurran (1989), se determinó la varianza de los índices de diversidad de Shannon-Wiener para los dos sistemas agroforestales, usando la siguiente formula:

$$
\operatorname{Var} H^{\prime}=\frac{\sum p_{i}\left(\ln p_{i}\right)^{2}-\left(\sum p_{i} * \ln p_{i}\right)^{2}}{N}+\frac{S-1}{2 N^{2}}
$$

Y mediante un sistema de cálculo de la " $\mathrm{t}$ " para comprobar las diferencias significativas entre muestras (Magurran, 1989), se usa la fórmula siguiente:

$$
t=\frac{H_{1}^{\prime}-H_{2}^{\prime}}{\left(\operatorname{Var} H_{1}^{\prime}+\operatorname{Var} H_{2}^{\prime}\right)^{1 / 2}}
$$

\section{RESULTADOS}

\section{Análisis taxonómico}

La s comunidades de 1 o s macroinvertebrados en los sistemas caracterizados comprenden tres Phyllum: Annelida, Mollusca y Arthropoda, siendo este último el que tuvo mayor presencia en el INAS; a este Phyllum pertenece las clases Insecta, Diplura, Diplopoda, Arachnida, Chilopoda y Malacostraca las cuales posee un gran número de ordenes
Donde $\mathrm{H}_{1}{ }_{1} \mathrm{y} \mathrm{H}_{2}$, son el valor de diversidad de 1 os sistemas INAS e ITAS respectivamente, de igual modo sucede con el valor de $\operatorname{Var}_{\mathrm{H}_{1}}$ y $\operatorname{Var} \mathrm{H}_{2}^{\prime}$.

Los grados libertad se calculan utilizando la ecuación:

$$
d f=\frac{\left(\operatorname{Var} H_{1}^{\prime}+\operatorname{Var} H_{2}^{\prime}\right)^{2}}{\left(\left(\operatorname{Var} H_{1}^{\prime}\right)^{2} / N_{1}\right)+\left(\left(\operatorname{Var} H_{2}^{\prime}\right)^{2} / N_{2}\right)}
$$

Haciendo uso de las tablas de la " $t$ " se revelan la diferencia estadística entre los dos sistemas agroforestales con respecto a la diversidad de macrofauna del suelo.

especialmente la clase Insecta así también fueron identificados los Phyllum Annelida y Mollusca con una menor presencia en el muestreo.

\section{Densidad de macrofauna registrada en el estudio}

La cuantificación de la abundancia por Phyllum en cada sistema agroforestal muestra mayores valores en el INAS (tabla 2), la mayor abundancia total (3694 individuos), en comparación al sistema agroforestal ITAS.

Tabla 2. Composición taxonómica de macrofauna edáfica colectada en sistemas agroforestales (INAS e ITAS)

\begin{tabular}{lccccrr}
\hline \multirow{2}{*}{ Phyllum } & \multicolumn{2}{c}{ Clase } & \multicolumn{2}{c}{ Orden } & \multicolumn{2}{c}{ Individuos colectados } \\
& INAS & ITAS & INAS & ITAS & INAS & \multicolumn{1}{c}{ ITAS } \\
\hline Annelida & 1 & 1 & 1 & 1 & 725 & 767 \\
Arthropoda & 6 & 6 & 18 & 15 & 2817 & 722 \\
Mollusca & 1 & 1 & 2 & 2 & 152 & 21 \\
$\quad$ Sumatoria & 8 & 8 & 21 & 18 & 3694 & 1510 \\
\hline
\end{tabular}

La mayor diversidad taxonómica estuvo presente en el sistema agroforestal INAS, aunque son considerados como valores muy bajos al relacionarlos con los sistemas naturales con cierto grado de conservación, como las selvas secundarias, que poseen gran riqueza taxonómica (Ararat et al., 2002; citados por Moran \& Alfaro, 2015). Esto se debe a que en los sistemas no intervenidos por el 
ser humano hay una menor y casi nula alteración del suelo, y la presencia de vegetación multiestratificada provee protección y fuentes de alimentos en diferentes grados de descomposición a los macroinvertebrados, humedad constante, así como mayores contenidos de materia orgánica (Moran \& Alfaro, 2015).

En el nivel de clases, fueron identificadas
8 en cada sistema agroforestal; siendo las más representativas la clase Insecta con un total de 2646 individuos, clitellata con 1492 individuos, diplura con 233 individuos y arachnida con 211 individuos, mientras las demás clases estuvieron representadas por un número menor de 199 individuos, conforme se aprecia en la figura 3 .

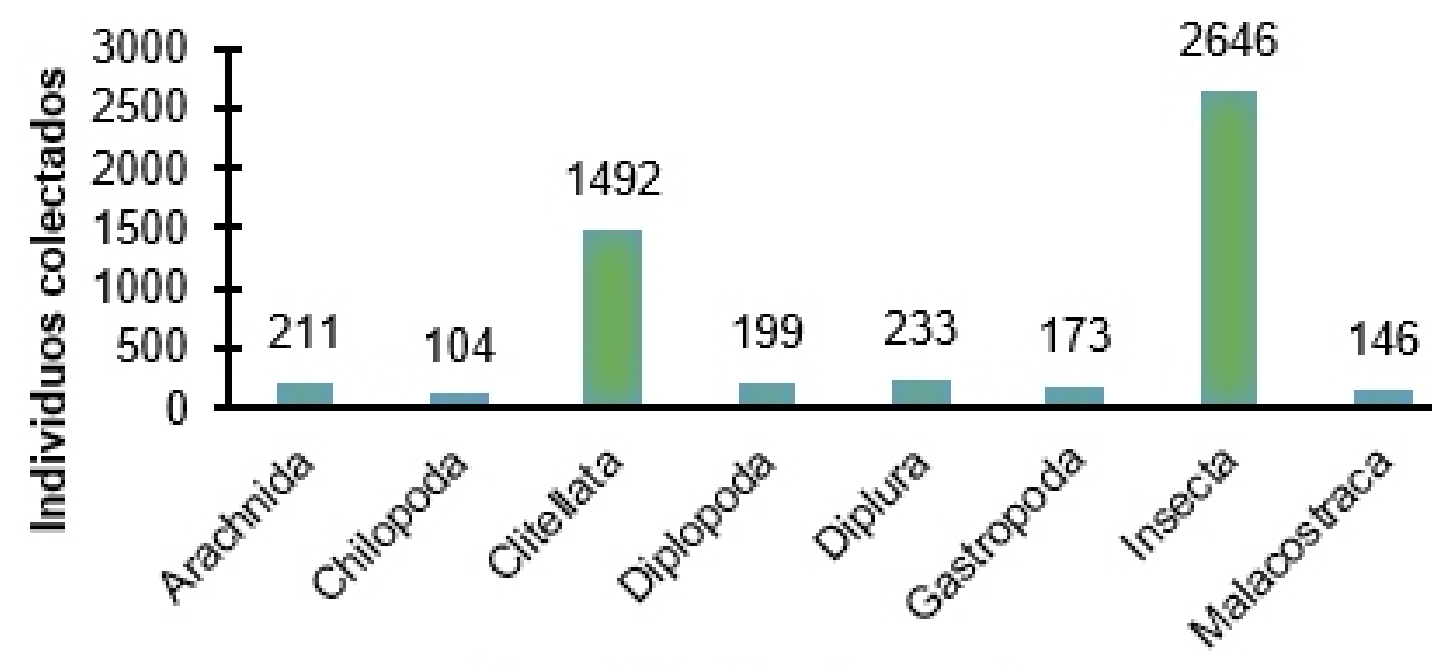

Clases identificadas en el muestreo

Figura 3. Individuos por clases de macrofauna edáfica identificadas en dos sistemas agroforestales del sector Choclino - San Martín.

La razón por la cual la clase Insecta tuvo mayor número de individuos es porque dentro de ellas se encuentran las termitas como más especie abundante. Esta especie pertenece a un grupo oportunista de rápida colonización, según Bandeira et al., (2003) citados por Moran \& Alfaro, (2015); estas están presentes en hábitat menos conservados y con algún nivel de degradación mientras que otros las señalan como las primeras especies colonizadoras en ambientes deforestados, con suficiente material leñoso remanente, en cuya descomposición intervienen como rol identificado (Cunha, 2006; citado por Moran y Alfaro, 2015).

\section{Biomasa de macrofauna registrada en el estudio}

En el caso de la biomasa el phylum artrópoda del sistema INAS tiene mayor valor en peso, con las clases Insecta, Diplura, Diplopoda, Arachnida, Chilopoda y Malacostraca las cuales poseen gran número de órdenes y por ende mayor peso, especialmente en la clase insecta. 
El peso de la biomasa por Phyllum en cada sistema agroforestal muestra mayores valores en el INAS (Tabla 3), la mayor biomasa total $\left(15.761148 \mathrm{~g} / \mathrm{m}^{2}\right)$, en comparación al sistema agroforestal ITAS $\left(8.141986 \mathrm{~g} / \mathrm{m}^{2}\right)$.

Tabla 3. Composición taxonómica y biomasa de macrofauna edáfica colectada en sistemas agroforestales (INAS e ITAS).

\begin{tabular}{lcccccc}
\hline \multirow{2}{*}{ Phyllum } & \multicolumn{2}{c}{ Clase } & \multicolumn{2}{c}{ Orden } & \multicolumn{2}{c}{ Peso $\left(\mathrm{g} / \mathrm{m}^{2}\right)$} \\
& INAS & ITAS & INAS & ITAS & INAS & ITAS \\
\hline Annelida & 1 & 1 & 1 & 1 & 2.818872 & 4.219397 \\
Arthropoda & 6 & 6 & 18 & 15 & 10.982743 & 3.852437 \\
Mollusca & 1 & 1 & 2 & 2 & 1.959533 & 0.070152 \\
& & & & Sumatoria & 15.761148 & 8.141986 \\
\hline
\end{tabular}

Een el nivel de clases, fueron identificadas 8 en cada sistema agroforestal; siendo las más representativas la clase Insecta con un total de $7.599111 \mathrm{~g} / \mathrm{m}^{2}$, Clitellata con $7.038269 \mathrm{~g} / \mathrm{m}^{2}$, Diplopoda con 3.134394 $\mathrm{g} / \mathrm{m}^{2}$, Arachnida con $2.317620 \mathrm{~g} / \mathrm{m}^{2} \mathrm{y}$ Gastropoda con $2.029685 \mathrm{~g} / \mathrm{m}^{2}$, mientras las demás clases estuvieron representadas por un número menor de $0.959209 \mathrm{~g} / \mathrm{m}^{2}$.

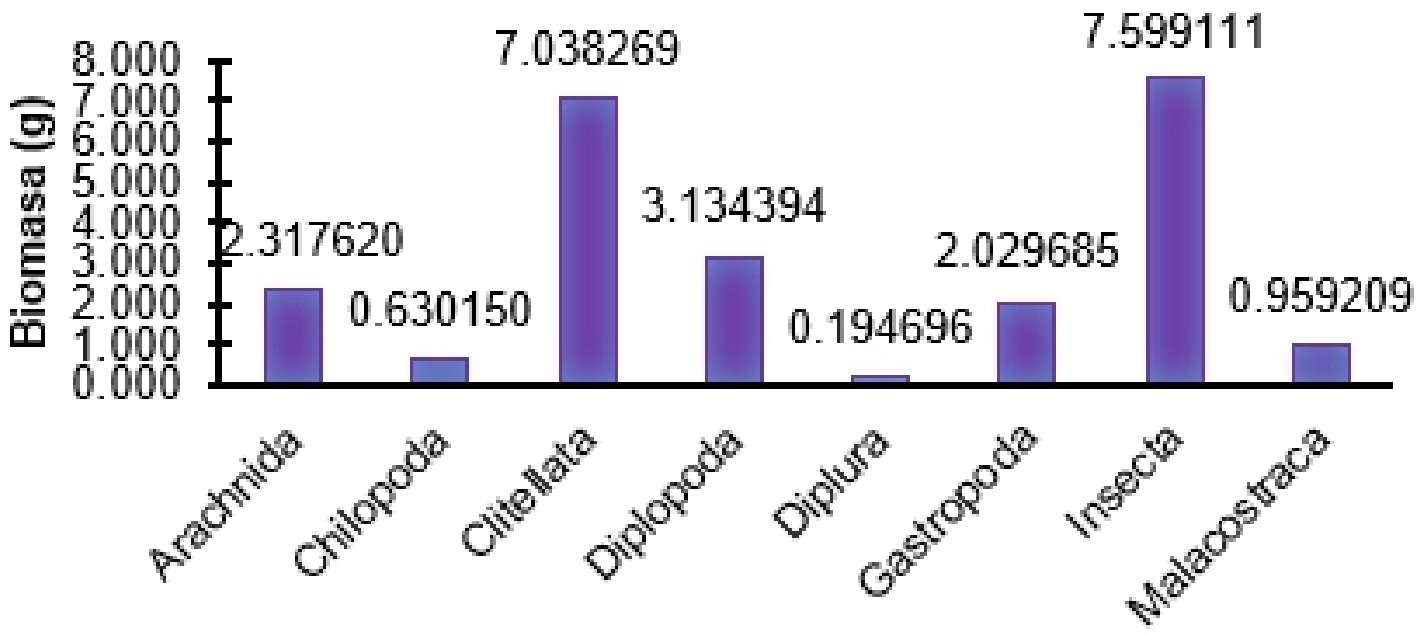

\section{Clases identificadas en el muestreo}

Figura 4. Biomasa por clases de macrofauna edáfica identificadas en dos sistemas agroforestales del sector Choclino - San Martín.

La clase Insecta tuvo mayor biomasa representada el peso en $\mathrm{g} / \mathrm{m}^{2}$ es porque dentro de ellas se encuentran las hormigas y termitas como especies abundantes y por ende mayor peso que implica el tamaño de dichas especies. Cunha, (2006); citado por Moran \& Alfaro, (2015) menciona que estas especies señalan como las primeras especies colonizadoras en ambientes deforestados, con suficiente material leñoso remanente, en cuya descomposición intervienen como rol identificado. 


\section{Curvas de abundancia- biomasa $\mathrm{ABC}$}

Respecto a la abundancia, los tres primeros órdenes del ranking destacados en densidad del INAS son: Hymenoptera (1193 ind./ $\left.\mathrm{m}^{2}\right)$, Haplotaxida (725 ind. $/ \mathrm{m}^{2}$ ) e Isoptera (685 ind. $\left./ \mathrm{m}^{2}\right)$; mientras que en la biomasa de macrofauna los órdenes sobresalientes son Haplotaxida (2.818872 $\mathrm{g} / \mathrm{m}^{2}$ ) seguido por Callipodida (2.603294 $\left.\mathrm{g} / \mathrm{m}^{2}\right)$ e Isoptera $\left(1.954043 \mathrm{~g} / \mathrm{m}^{2}\right)$.

De acuerdo a Martínez (2012) que representa en la figura 1 , y basándose en la estimación del estadístico W, obtenida a través de la comparación de las curvas abundancia-biomasa (ABC), en el sistema agroforestal INAS, se puede deducir que la comunidad esta moderadamente perturbada (figura 5).

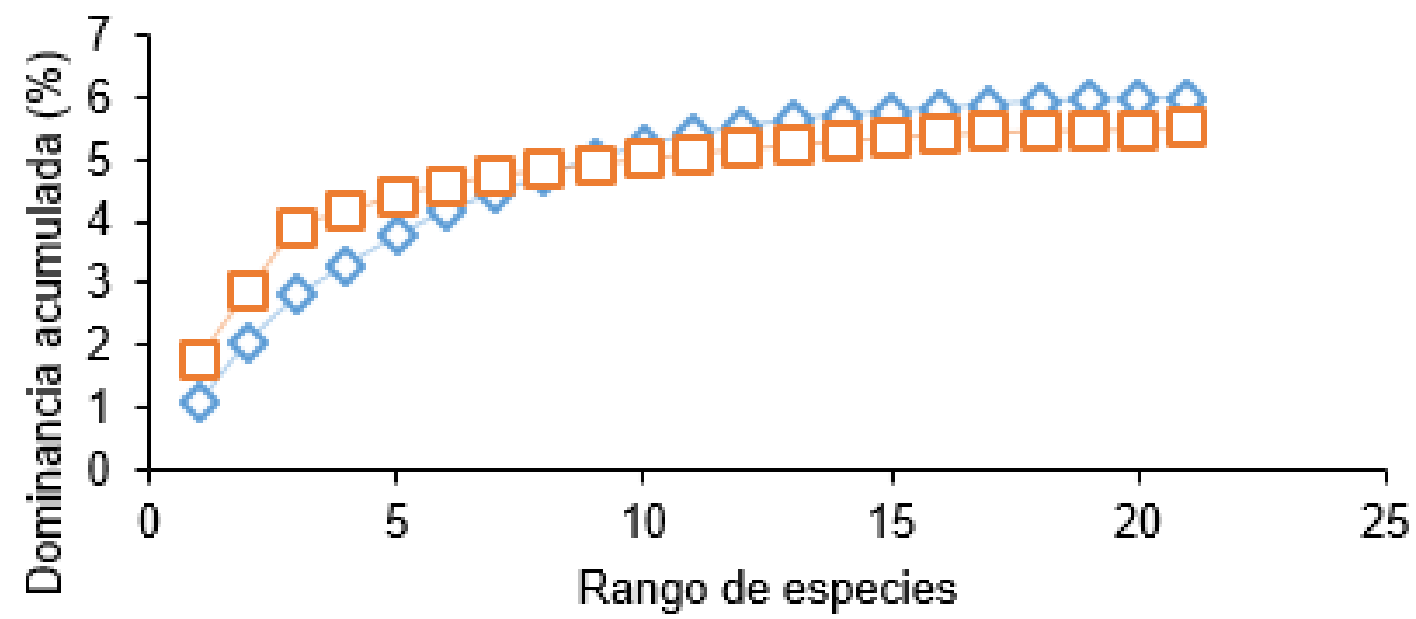

\section{$\multimap$ Biomasa $\quad \square-$ Abundancia}

Figura 5. Curva de Abundancia - biomasa $\mathrm{ABC}$ de la macrofauna del suelo en el INAS Choclino.

La representación del área entre las curvas Abundancia -Biomasa es el valor obtenido de la formula Warwick y Clarke, (1994); citados por Martínez (2012), es de $\mathrm{W}=5.32907 \times 10^{-18}$, el valor positivo es por situaciones caracterizadas a un mínimo stress.

Respecto a la abundancia los tres primeros órdenes del ranking destacados en densidad del ITAS son: Haplotaxida (767 ind. $/ \mathrm{m}^{2}$ ), Hymenoptera (209 ind. $/ \mathrm{m}^{2}$ ) y Coleoptera (85 ind. $\left./ \mathrm{m}^{2}\right)$; de acuerdo a la biomasa de macrofauna los órdenes sobresalientes son Haplotaxida (4.219397 $\left.\mathrm{g} / \mathrm{m}^{2}\right)$ seguido de Araneae $\left(2.115336 \mathrm{~g} / \mathrm{m}^{2}\right)$ e Hymenoptera $\left(0.490678 \mathrm{~g} / \mathrm{m}^{2}\right)$.

De acuerdo a Martínez (2012) que representa en la figura 1 , y basándose en la estimación del estadístico $\mathrm{W}$, obtenida a través de la comparación de las curvas abundancia-biomasa (ABC), en el sistema agroforestal ITAS, se puede deducir que la comunidad esta moderadamente perturbada (figura 6). 


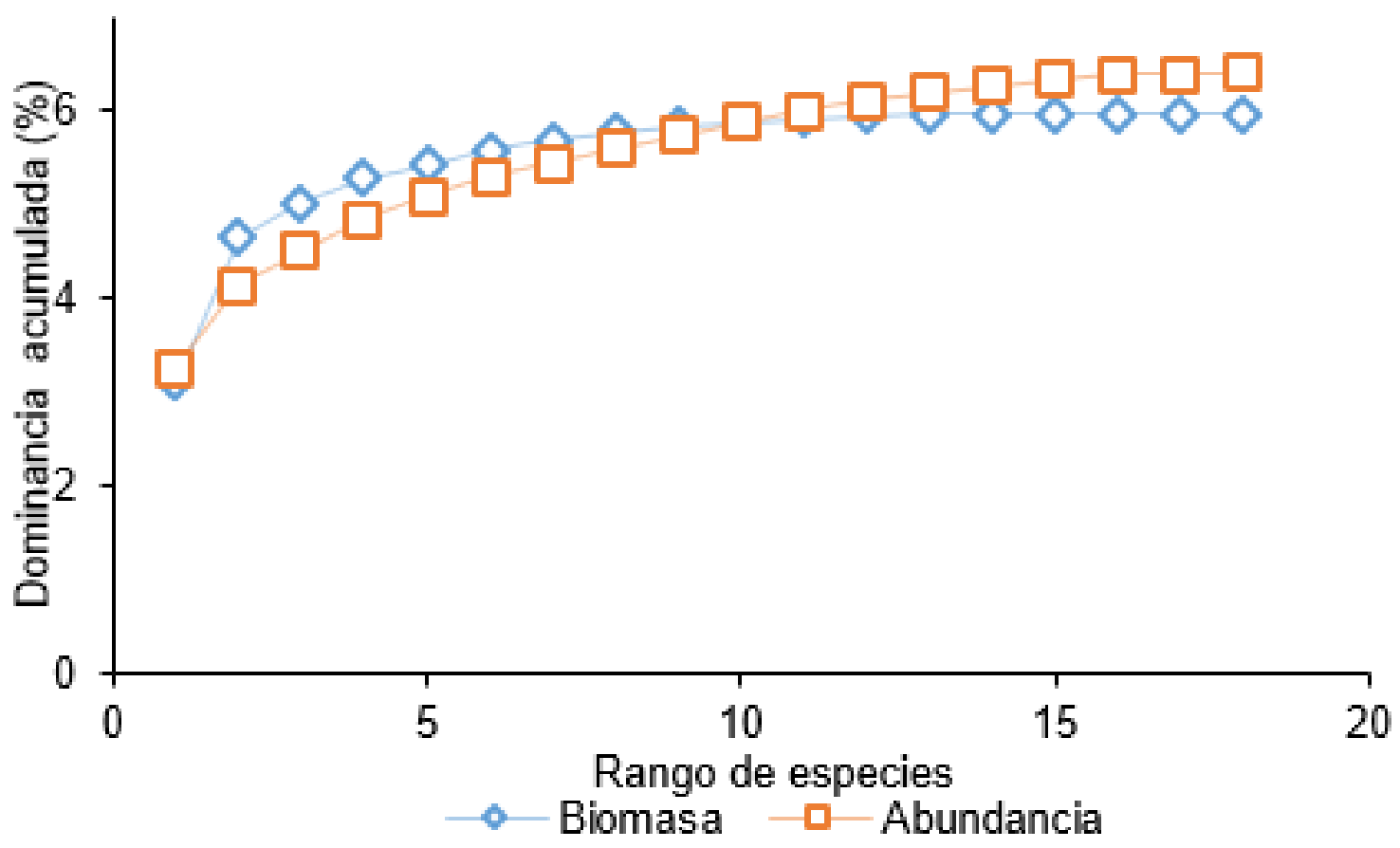

Figura 6. Curva de Abundancia - biomasa ABC de la macrofauna del suelo en el ITAS Choclino.

La representación del área entre las curvas Abundancia - Biomasa es el valor obtenido de la formula Warwick y Clarke, (1994); citados por Martínez (2012) es de $\mathrm{W}=-2.22045 \times 10^{-18}$, el valor negativo es por situaciones caracterizadas por un fuerte stress.

\section{Atributos comunitarios de los sistemas agroforestales}

Respecto a los atributos comunitarios de la macrofauna en los dos sistemas agroforestales en el sector el Choclino, el cuadro 4 nos muestra los resultados obtenidos de la macrofauna por el índice de diversidad de Shannon - Wienner $\left(\mathrm{H}^{\prime}\right)$, el índice de Equidad para la determinar la uniformidad entre los sistemas; a raíz de los resultados del índice antes mencionado y la aplicación de la formula se obtuvo el índice de Equidad para los dos sistemas agroforestales, tal como se puede apreciar en el tabla 4.

Tabla 4. Atributos comunitarios de los sistemas agroforestales.

\begin{tabular}{ccc}
\hline Sistema agroforestal & Shannon-Wiener H' & Equidad $E$ \\
\hline INAS & 2.114389 & 0.694489542 \\
ITAS & 1.874392 & 0.648495260 \\
\hline
\end{tabular}

Análisis estadístico del índice de diversidad

Con la base de datos de identificación, número de especies y número de individuos se obtuvo el índice de diversidad de Shannon $\left(\mathrm{H}^{\prime}\right)$ para cada sistema agroforestal INAS e ITAS.

Del desarrollo de la fórmula para determinar la varianza de los índices de 
diversidad de Shannon-Wiener de los dos sistemas agroforestales:

$$
\begin{array}{ll}
\operatorname{Var} H_{\mathrm{INAS}}^{\prime}=\frac{5.844116-0.431307}{3614}+\frac{21-1}{2 * 3694^{2}} & \operatorname{Var} H_{\mathrm{INAS}}^{\prime}=0.00146603 \\
\operatorname{Var} H^{\prime}{ }_{\mathrm{INAS}}=\frac{5.36910-0.32214}{1510}+\frac{18-1}{2 * 1510^{2}} & \operatorname{Var} H_{\mathrm{INAS}}^{\prime}=0.003346609
\end{array}
$$

Cálculo de la " $t$ " para comprobar las diferencias significativas entre muestras, mediante el desarrollo de la siguiente formula:

$$
\mathrm{t}=\frac{2.11439-1.87439}{(0.00146603+0.00334609)^{1 / 2}} \quad \mathrm{t}=3.45969
$$

Calculo del grado libertad:

$$
d f=\frac{(0.00146603+0.00334609)^{2}}{\left((0.00146603)^{2} / 3694\right)+\left((0.00334609)^{2} / 1510\right)} \quad d f=2895.79
$$

Observando y comparando los datos en la tabla " $t$ " nos revela que los dos sistemas a g r o foresta 1 e s s o n m u y significativamente distintos $(\mathrm{P}<0.001)$ en términos de diversidad de macrofauna del suelo. El sistema INAS es, por lo tanto, más diverso que el sistema ITAS.

\section{DISCUSIÓN Y CONCLUSIONES}

\section{Análisis taxonómico e indicadores agregados}

L a s comunidade s d e 1 o s macroinvertebrados en los sistemas caracterizados comprenden tres Phyllum: Annelida, Mollusca y Arthropoda, siendo este último el que tuvo mayor presencia en el INAS; a este Phyllum pertenece las clases Insecta, Diplura, Diplopoda, Arachnida, Chilopoda y Malacostraca las cuales posee un gran número de ordenes especialmente la clase Insecta; así también fueron identificados los Phyllum Anelida y Mollusca con una menor presencia en el muestreo. La mayor diversidad taxonómica estuvo presente en el sistema agroforestal INAS, aunque son considerados como valores muy bajos al relacionarlos con los sistemas naturales con cierto grado de conservación, como las selvas secundarias, que poseen gran riqueza taxonómica (Ararat et al., 2002; citados por Moran \& Alfaro, 2015). Esto se debe a que en los sistemas no intervenidos por el ser humano hay una menor y casi nula alteración del suelo, y la presencia de vegetación multiestratificada provee protección y fuentes de alimentos en diferentes grados de descomposición a los macroinvertebrados, humedad constante, así como mayores contenidos de materia orgánica (Moran \& Alfaro, 2015).

En el nivel de clases, fueron identificadas 8 en cada sistema agroforestal; siendo el INAS con mayor número de individuos 3694 ind. $/ \mathrm{m}^{2}$ y biomasa $15.761148 \mathrm{~g} / \mathrm{m}^{2}$; 
en comparación con el estudio realizado por Linares (2009), los resultados son mayores, obteniendo que en el suelo con cobertura forestal, se tiene la mayor cantidad de individuos representantes de la mesofauna con 16,304 ind. $/ \mathrm{m}^{2}$, esto indica que la macro fauna del suelo es un componente principal dentro de los ecosistemas ya que participan efectivamente en el reciclaje de los nutrientes del suelo, favoreciendo al crecimiento de las especies.

La biomasa de la macrofauna del suelo fue mayor en el INAS con $15.761148 \mathrm{~g} / \mathrm{m}^{2}$, mientras que en el ITAS presentó $8.141986 \mathrm{~g} / \mathrm{m}^{2}$; Brown et al. (2001) mencionan que una biomasa mínima de $30 \mathrm{~g} / \mathrm{m}^{2}$ de lombrices de tierra puede tener importantes consecuencias sobre el suelo y la productividad vegetal, esta biomasa se encontró en los policultivos arbóreos y sistemas naturales principalmente.

Referente a la curva de dominancia (abundancia-biomasa) ABC calculada para ambos sistemas agroforestales, las comunidades de macrofauna del suelo presentaron una moderada perturbación con forme se muestra en la figura 2 , con un $\mathrm{W}=5.32907 \times 10^{-18}$ para el INAS, dicho valor positivo es por situaciones caracterizadas a un mínimo stress y en el caso del ITAS se obtuvo un $\mathrm{W}=$ $2.22045 \times 10^{-18}$, el valor negativo es por situaciones caracterizadas a un fuerte stress; así Martínez (2012) menciona que durante la temporada 2003, la comunidad presentó una moderada perturbación (se entrecruzan las curvas), mientras tanto en
2004 y 2005 presentó alta perturbación (curva de abundancia por encima de la curva de biomasa).

\section{Atributos comunitarios de los sistemas agroforestales}

Los atributos comunitarios de la macrofauna del suelo en los dos sistemas agroforestales del sector Choclino nos muestra los resultados obtenidos por el índice de diversidad de Shannon Wienner $\left(\mathrm{H}^{\prime}\right)$ en el que el sistema INAS obtuvo un valor de 2.11439 nats/ind. que según Ramirez (2006) es alta; mientras que el sistema ITAS obtuvo un valor de 1.87439 nats/ind. presentando una diversidad media.

Respecto al índice de Equidad (E) que determina la uniformidad, los valores fueron 0.694489542 especies para el sistema INAS y 0.648495260 especies para el ITAS, según Magurran (1989) la diversidad para ambos sistemas agroforestales seria de un nivel medio.

\section{Análisis estadístico del índice de diversidad}

El análisis de índice, es decir del cálculo y desarrollo estadístico del índice de Shannon-Wiener para comprobar las diferencias de diversidad entre los sistemas, nos revela que los dos sistemas a groforestales son mu significativamente distintos $(\mathrm{P}<0.001)$ en términos de diversidad de macrofauna del suelo conforme se muestra en los resultados; el sistema INAS es, por lo tanto, más diverso que el sistema ITAS. Al respecto Batten (1976) citado por Magurran (1989) menciona que en 
un estudio se registró la riqueza de especies de pájaros y su abundancia en diversos bosques nativos y en plantaciones de coníferas de Killarney, Irlanda. La finalidad, en parte, era determinar si las plantaciones de coníferas están empobrecidas respecto a los bosques endémicos. Obteniendo que son significativamente distintos, por lo que el bosque nativo es, más diverso que la plantación de coníferas.

En tal sentido se concluye que:

1. Los suelos del sistema agroforestal nativo - INAS presentó el doble de individuos por metro cuadrado, en comparación a la cantidad de individuos recolectados en el sistema agroforestal tradicional - ITAS.

2. La biomasa de la macrofauna del suelo fue mayor en el INAS, mientras que en el ITAS presentó la mitad de lo reportado por el sistema nativo.

3. La curva de dominancia (abundanciabiomasa) ABC, calculada para ambos sistemas agroforestales, presentaron una moderada perturbación en las comunidades de macrofauna del suelo

3. Con respecto a los atributos comunitarios de la macrofauna del suelo en el INAS se obtuvo un alto índice de diversidad de ShannonWiener y en el ITAS un índice medio.

4. La equidad de ambos sistemas agroforestales tienen un valor medio.

5. Los dos sistemas agroforestales en términos de diversidad de macrofauna del suelo son muy significativamente distintos $(\mathrm{P}<0.001)$.

\section{REFERENCIAS BIBLIOGRÁFICAS}

Anderson J. \&, Ingranm, J. (1993). Tropical soil biology and fertility: A Handbook of methods. Segunda edición. Wallingford - United Kingdom, CAB international. 237 p.

Brown, G., Fragoso, C., Barois, I., Rojas, P., Patrón, J., Bueno, J., Moreno, A., Lavelle, P., Ordaz, V., Rodríguez, C. (2001). Diversidad y rol funcional de la macrofauna edáfica en los ecosistemas tropicales mexicanos. Instituto de Ecología. Xalapa, México. Acta Zoológica Mexicana (nueva serie). Número Especial 1: 79-110.

Cabrera, G. (2012). La macrofauna edáfica como indicador biológico del estado de conservación/perturbación del suelo. Resultados obtenidos en Cuba. Pastos y Forrajes, Vol. 35 (4): $\begin{array}{llllllll}3 & 4 & 9 & - & 3 & 6 & 4\end{array}$. http://www.redalyc.org/articulo.oa?i $\mathrm{d}=269125514007$.

Campos, I. (2010). La macrofauna de suelo como indicador biológico en diferentes usos de suelos agrícolas, en el caserío los Milagros-Aucayacu. Tesis Ing. Recursos Naturales Renovables. Tingo María, Perú, Universidad Nacional Agraria de la Selva. 109 p.

Decaens, T., Lavelle, P., Jiménez-Jaen, J.J., Escobar, G., Rippstein, G. (1994). Impact of land management 
on soil macrofauna in the oriental llanos of Colombia. European Journal Soil Biology, Vol.30 (4): 157-168.

Linares, D. (2009). Macrofauna del suelo en diferentes sistemas de uso de la tierra en el Parque Nacional Tingo María, Huánuco, Perú. 78 p.

Magurran, A. (1989). Diversidad ecológica y su medición. Barcelona, España. Ediciones Vedrà. 200 p.

Martínez, A. (2012). Estructura y distribución de la comunidad íctica acompañante en la pesca del camarón (Golfo de Tehuantepec. Pacifico oriental, México). Tesis doctoral. Universidad de Barcelona. México. $635 \mathrm{p}$.

Moran, J., Alfaro, F. (2015). Diversidad de macrofauna edáfica en dos sistemas de manejo de Moringa oleifera Lam. (Marango) en la finca Santa Rosa, UNA. Tesis ingeniería forestal. Managua, Nicaragua. Universidad Nacional Agraria. 46 p.

Moreno, C. E. 2001. Métodos para medir la biodiversidad. M\&T-Manuales y Tesis SEA, vol.1. Zaragoza, $84 \mathrm{pp}$.

Ñique, M. (2010). Biodiversidad: Clasificación y Cuantificación. Universidad Nacional Agraria de la Selva. Tingo María, Perú. 17 p.

Odum, E. 1972. Ecología del suelo. México. Editorial Interamericana. $295 \mathrm{p}$.

ONERN, 1976. Mapa ecológico del Perú, guía explicativa. Lima - Perú. http://library.wur.nl/isric/fulltext/isr icu_i00006671_001.pdf

Pashanasi, B. 2001. Estudio cuantitativo de la macrofauna del suelo en diferentes sistemas de uso de la tierra en la Amazonía peruana. Folia Amazónica Vol. 12(1-2): 75-97.

Ramirez, A. (2005). Ecología aplicada: diseño y análisis estadístico. Fundación universal de Bogotá. Santa Fe de Bogotá, Colombia. 327 p.

Ramirez, A. (2006). Ecología. Métodos de muestreo y análisis de poblaciones y comunidades. Bogotá. Pontificia Universidad Javeriana. $271 \mathrm{p}$.

\section{CORRESPONDENCIA:}

Ing. Shirley Ornella Castillo Pérez E-mail: shirone1316_8@hotmail.com 